\title{
Chromosome painting and its applications in cultivated and wild rice
}

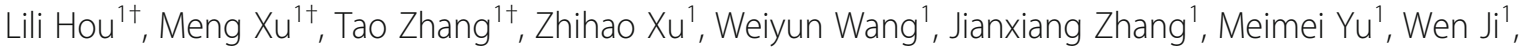 \\ Cenwen Zhu', Zhiyun Gong', Minghong Gu', Jiming Jiang ${ }^{2}$ and Hengxiu Yu ${ }^{*^{*}}$ (i)
}

\begin{abstract}
Background: The chromosome-specific probe is a fundamental tool of chromosome painting and has been commonly applied in mammalian species. The technology, however, has not been widely applied in plants due to a lack of methodologies for probe development. Identification and labeling of a large number of oligonucleotides (oligos) specific to a single chromosome offers us an opportunity to establish chromosome-specific probes in plants. However, never before has whole chromosome painting been performed in rice.
\end{abstract}

Results: We developed a pooled chromosome 9-specific probe in rice, which contains 25,000 oligos based on the genome sequence of a japonica rice (Oryza sativa L., AA, $2 n=2 x=24$ ). Chromosome 9 was easily identified in both japonica and indica rice using this chromosome 9-painting probe. The probe was also successfully used to identify and characterize chromosome 9 in additional lines of O. sativa, a translocation line, two new aneuploids associated with chromosome 9 and a wild rice (Oryza eichingeri A. Peter, CC, $2 n=2 x=24)$.

Conclusion: The study reveals that a pool of oligos specific to a chromosome is a useful tool for chromosome painting in rice.

Keywords: Oryza sativa, Oryza eichingeri, FISH, Chromosome painting, Oligonucleotide, Chromosome variation

\section{Background}

Chromosome painting $(\mathrm{CP})$ is a technique of fluorescence in situ hybridization (FISH), which offers a powerful method for detection of specific chromosome regions or entire chromosomes based on chromosome-specific probes $[1,2]$. In the past few decades, this technique has been primarily used to study human and animal chromosomes for diagnosing chromosome abnormalities, investigating chromosome rearrangements during evolution, and constructing ancestral karyotypes [3-7]. It has also been applied to molecular cytotaxonomy [8-11]. Chromosome painting probes in these cases were derived from flow-sorted or micro-dissected chromosomes followed by degenerate oligonucleotide-primed PCR amplification [12-14].

\footnotetext{
* Correspondence: hxyu@yzu.edu.cn

${ }^{\dagger}$ Lili Hou, Meng Xu and Tao Zhang contributed equally to this work. 'Key Laboratory of Plant Functional Genomics of Ministry of Education/ Jiangsu Key Laboratory of Crop Genetics and Physiology/Co-Innovation Center for Modern Production Technology of Grain Crops, Yangzhou University, Yangzhou 225009, China

Full list of author information is available at the end of the article
}

For most plant genomes, DNA probes prepared from flow-sorted or micro-dissected plant chromosomes failed to yield satisfactory results due to the presence of vast amounts of repetitive DNA that cannot be efficiently eliminated or blocked in FISH experiments [15-18]. To overcome such difficulties, a new FISH technique using large-insert DNA clones [bacterial artificial chromosomes (BACs) or yeast artificial chromosomes (YACs)] was developed for some plant species with small genomes. Fransz et al. used several YACs to paint specific regions of Arabidopsis thaliana L. chromosome 4 [19]. Contiguous BACs were also grouped as probes to paint an individual chromosome in A. thaliana [20] and Brachypodium distachyon L. [15]. This chromosome painting technique is a powerful tool to study genome duplication, chromosomal rearrangement, and evolution in species associated with $A$. thaliana and $B$. distachyon [21-25]. However, this technique requires ordered BAC contigs that cover the entire genome of a plant species. More importantly, it relies on the fact that both $A$. thaliana and $B$. distachyon have relatively small

(c) The Author(s). 2018 Open Access This article is distributed under the terms of the Creative Commons Attribution 4.0 International License (http://creativecommons.org/licenses/by/4.0/), which permits unrestricted use, distribution, and reproduction in any medium, provided you give appropriate credit to the original author(s) and the source, provide a link to the Creative Commons license, and indicate if changes were made. The Creative Commons Public Domain Dedication waiver (http://creativecommons.org/publicdomain/zero/1.0/) applies to the data made available in this article, unless otherwise stated. 
genomes. Most of the selected BACs contain almost exclusively single- or low-copy sequences.

Another successful chromosome painting method is based on PCR amplification to pool a large number of single-copy sequences. Lou et al. applied this method and obtained seven gene pool probes with sizes above $2 \mathrm{~kb}$ and an interval less than $300 \mathrm{~kb}$ to successfully paint each pachytene chromosome pair in Cucumis sativus L. [26]. A cucumber karyotype was also constructed based on these chromosome-specific probes and a comparative chromosome map of a region on chromosome 4 was constructed for both cucumber and melon. Unfortunately, amplification of a large number of PCR products that cover an entire chromosome is especially difficult and time consuming for plant species with large and complex genomes.

Sequence availability of the target DNA and massive parallel synthesis techniques make it possible to produce complex oligonucleotide (oligo) library that consist of thousands of independent oligos. Moreover, this kind of probes has been used successfully as FISH probes in mammalian and Drosophila species [27-29]. Han et al. first applied this technique in plants [30]. Oligo library specific to a single chromosome of cucumber was identified using a bioinformatics pipeline and then oligos were massively synthesized de novo in parallel. The synthesized oligos were amplified and labeled with biotin or digoxigenin, and were used as probes in FISH. They developed three different probes with each containing 23,000-27,000 oligos. These probes spanned 8.3-17 Mb of DNA on targeted cucumber chromosomes and had a density of 1.5-3.2 oligos per kilo base pairs. These oligo probes can be used to track homeologous chromosome pairing in early meiotic stages and examine the chromosome pairing behavior between homeologous chromosomes of cucumber. Divergence between Cucumis melo L. and an African Cucumis species were identified by chromosome painting using an oligo library and combining rDNA distribution patterns [31]. This technique was also used successfully to study the sex chromosomes in Populus tomentosa Carr. and Populus deltoids March [32]. Braz, et al. developed a bar code containing 54,672 oligos to label chromosomes from both diploid and polyploid potato species. This probe can also identify the homeologous chromosomes among distantly related species of Solanum L., including tomato and eggplant [33].

The genus Oryza L. consists of more than 20 species, including about 20 wild Oryza species and two cultivated species. The genomes of the wild Oryza species can be classified into 10 distinct groups (A, B, BC, C, CD, D, E, F, G, HJ and HK). Except O. brachyantha A. Chev. \& Roehr. (F genome), all species in the genus Oryza are grouped into four main species complexes: sativa, officinalis, ridleyi and meyeriana, based on classical taxonomy, isozyme, RFLP and gene sequences. Nine species with $B, B C, C, C D$ and $E$ genomes were grouped to the officinalis complex [34-36]. The two cultivated species, O. sativa L. and O. glaberrima Steud. with 24 chromosomes are grouped in A genome. Two sub species, japonica and indica were identified as $O$. sativa based on their morphology and growth habitats $[35,37,38]$. They were likely domesticated from a specific wild rice population of O. rufipogon W. Griffith (A genome) [39, 40]. Oryza officinalis Wall. ex Watt and O. eichingeri A. Peter are diploid species with $\mathrm{C}$ genomes $(2 n=2 x=24)$. Oryza officinalis is considered to be the basic genome of the officinalis complex and is considered to be similar to the A genome of $O$. sativa $[35,41]$. The wild rice with $C$ genomes is an important resource of valuable traits for the improvement of cultivated rice, such as disease and insect resistances [42-45]. But there is less research on O. eichingeri. Oryza sativa is one of the most important crops and an excellent model plant for monocotyledonous plants because it possesses a small genome that has also been completely sequenced. Despite the fact that the genomic sequence of a japonica variety, Nipponbare, has been available for a long time [46], no whole-chromosome painting experiment has been performed in any rice species. To study the possibility of application of oligo probes in O. sativa and examine the differences between O. sativa and O. eichingeri, we developed a library containing 25,000 oligos for identification of chromosome 9 in rice. This probe generated bright and chromosome-specific FISH signals in O. sativa. We used it to confirm a translocation between chromosome 9 and 11, as well as identify two new aneuploids associated with chromosome 9. This chromosome 9-specific oligo library could also be useful to trace chromosome 9 of $O$. eichingeri.

\section{Results \\ Establishment of a chromosome-specific oligonucleotide set}

To develop the chromosome 9-specific painting probe, we identified all non-overlapping oligos that are unique to chromosome 9 of Nipponbare using the bioinformatics pipeline developed by Han et al. with some modification [30]. Oligos with homology to repetitive DNA sequences or to sequences located on other chromosomes were eliminated. The oligo set containing 25,000 oligos was selected basing on the density of two oligos per kilobase, covering the entire length of chromosome 9 of Nipponbare. Sequence data of the oligo library can be found in the Additional file 1.

Sensitivity and reliability of oligo probe for a japonica rice For testing the sensitivity and reliability of the oligo probe for chromosome 9, a digoxigenin-labeled oligo 
set and a biotin-labeled $45 \mathrm{~S}$ ribosomal RNA gene(45S rDNA)probe were hybridized to interphase and prometaphase chromosomes which were prepared from root tips of Nipponbare plants. The $45 \mathrm{~S}$ rDNA signal is typically located at the telomeric region on chromosome 9 in japonica rice [47]. Both probes produced bright FISH signals on interphase nuclei and prometaphase chromosomes (Fig. 1a and b). Signal of the chromosome 9-specific probe occupied two separate domains on interphase nuclei (Fig. 1a). As expected, the FISH signal from the oligo probe (red) nearly uniformly covered the entirety of chromosome 9 without signal gaps. No cross-hybridization signal was detected on any other chromosomes. The 45S rDNA signals (green) were detected at the ends of the short arms of chromosome 9. To demonstrate the convenience of the oligos for chromosome painting in meiosis, the chromosome 9 specific probe was also hybridized to meiotic chromosomes of Nipponbare (Fig. 1c, d, e and f). During premeiotic interphase, two painted chromosome 9 were detected. They were separated from each other (Fig. 1c). However, during the late zygotene stage, the two painted chromosome 9 aligned very well (Fig. 1d). Fully synapsed chromosome 9 was observed at the pachytene stage (Fig. 1e).
However, only one chromosome harbored the FISH signal in the microspore, indicating that the chromosome number was halved in the nucleus after meiosis (Fig. 1f). In addition, the intensity of the FISH signal on the zygotene and pachytene chromosomes was not as strong as that on mitotic prometaphase chromosomes. These results confirm that chromosome 9-specific probe is sensitive and reliable for identifying both mitotic and meiotic chromosomes of rice in $\mathrm{CP}$ experiments.

\section{Delimitation of chromosome 9 of an indica rice and identification of chromosome translocation using a chromosome 9-specific oligo probe}

To test if the chromosome 9-specific probe selected based on a japonica rice would work for indica rice and confirm the chromosome rearrangement in a translocation line derived from an indica rice Zhongxian 3037 [48], the chromosome 9-specific oligo library and 5S ribosomal RNA genes(5S rDNA)were used as probes in the FISH experiment. The $5 \mathrm{~S}$ rDNA locates on the short arm of rice chromosome 11 close to the centromere [49]. In normal mitotic prometaphase cells of Zhongxian 3037, 5S rDNA signals can be detected on two chromosomes. However, during mitotic prometaphase, the

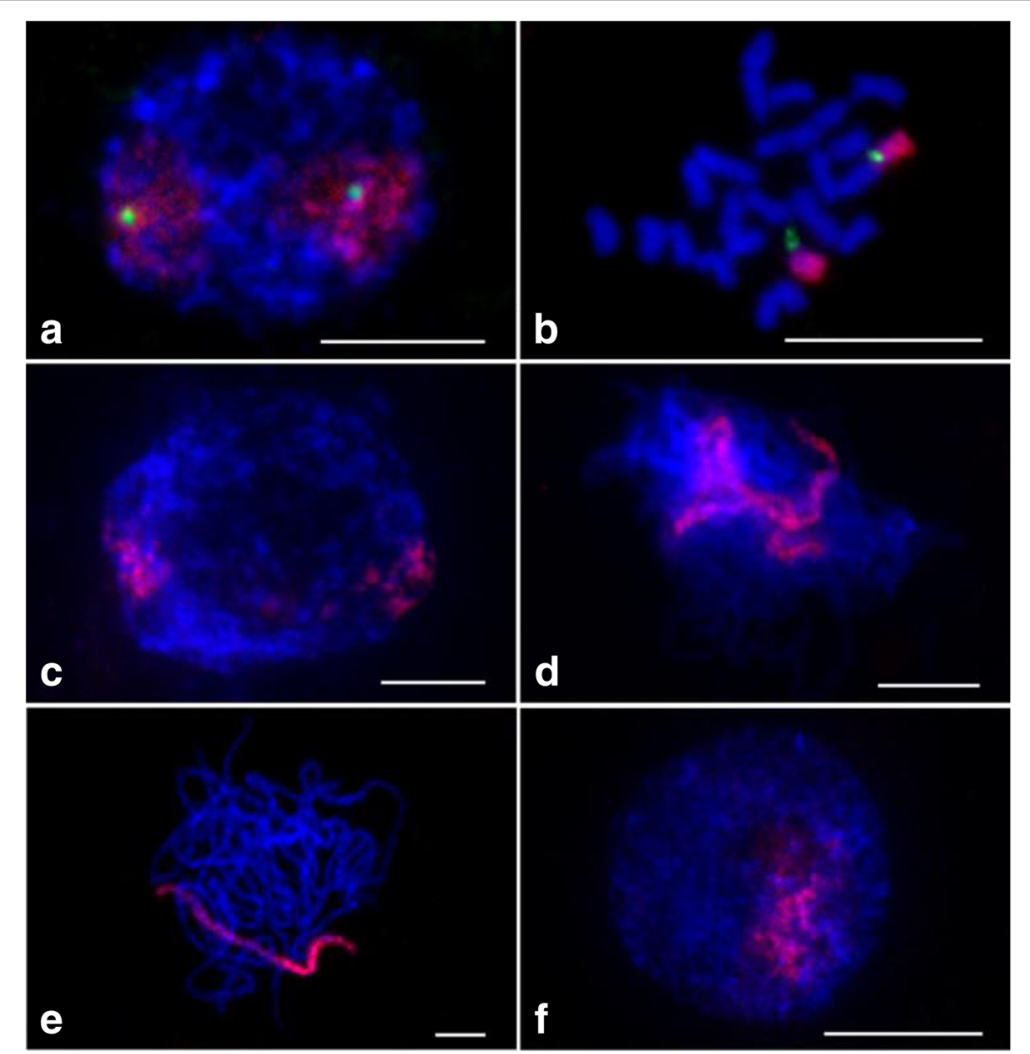

Fig. 1 FISH using the chromosome 9-specific oligo library and 45S rDNA probes on chromosomes of Nipponbare. The chromosome 9-specific (red) and 455 rDNA probes (green) fluorescing in chromosomes in the (a) interphase, (b) prometaphase, (c) premeiotic interphase, (d) zygotene, (e) pachytene and (f) microspore. Chromosomes were stained with DAPI. Bars, $5 \mu \mathrm{m}$ 
signals of both the digoxigenin-labeled chromosomes 9specific (red) and biotin-labeled 5S rDNA probes (green) were observed closely adjacent to each other on four chromosomes in the translocation line (Fig. 2a and b). Furthermore, the translocation chromosomes with the long arms of chromosome 11 had brighter signals of $5 \mathrm{~S}$ rDNA. These results indicate that a reciprocal translocation had happened between chromosomes 9 and 11 and the translocation breakpoint was likely located within the $5 \mathrm{~S}$ rDNA array on chromosome 11. During the pachytene stage, both probes produced bright FISH signals on two bivalents of this mutant (Fig. 2c and d). Chromosomes 9 (green) and 5S rDNA (red) signals were closely adjacent to each other on both bivalents, which also confirmed that the translocation occurred between chromosome 9 and 11. Thus chromosome 9-specific oligo probe selected based on a japonica rice also performed well in an indica rice.

To further characterize the translocation chromosome, the 155-bp CentO repeat, which is specific to centromeres of rice chromosomes [50] and the chromosome 9-specific probe were used for FISH. Chromosome 9 is an acrocentric chromosome and the content of CentO in chromosome 11 is about two times that of chromosome 9 in Zhongxian 3037 [51]. In the translocation stock, a strong CentO signal (green) was detected on the longer translocation chromosomes (Fig. 2e and f). CentO signal was not consistently detected on the shorter translocation chromosomes during mitotic prometaphase (Fig. 2e and f). However, clear CentO signals were detected on both the long and short translocation chromosomes at the pachytene stage (Fig. $2 \mathrm{~g}$ and h). Interestingly, two separate flourescing blocks of CentO signals were observed on the longer translocation bivalent. The CentO signal was weaker on the shorter translocation bivalent, indicating that the shorter translocation chromosomes harbored the smaller parts of the centromeres of chromosome 9. The longer translocation chromosomes were designated as 9 L.11 L because they harbored the long arms of chromosome 9 (red) and chromosome 11 (blue). Whereas the shorter translocation chromosomes, designated as 9S.11S had the short arms of chromosome 9 and chromosome 11. Taken together, the breakpoint in chromosome 9 occurred in the CentO array, which resulted in two CentO arrays; the large array was received by $11 \mathrm{~L} .9 \mathrm{~L}$ and the small one was received by $11 \mathrm{~S} .9 \mathrm{~S}$ (Fig. 2i). The breakpoint in chromosome 11 occurred in the middle of the $5 \mathrm{~S}$ rDNA locus, which resulted in $5 \mathrm{~S}$ rDNA signals on both the 11 L.9 L and 11S.9S chromosomes (Fig. 2i).

\section{Characterization of rice aneuploids associated with chromosome 9}

To identify and characterize aneuploids associated with chromosome 9, we selected putative aneuploid plants with different phenotypes compared to the trisomic 9 and Zhongxian 3037 from progenies of a rice trisomic 9 $(2 \mathrm{n}=25$, with three copies of chromosome 9). To investigate the potential chromosomal mutation in these plants, 45S rDNA, CentO and the chromosome 9-specific oligo probes were used in FISH analyses. The $45 \mathrm{~S}$ rDNA signal is typically located at telomeric regions on both chromosome 9 and 10 in Zhongxian 3037 [47]. The mutant line, YN6077 contained 25 chromosomes, including a pair of normal chromosome 9 and an additional short chromosome. This short chromosome bore a chromosome 9-specific signal (red), and the 45S rDNA signal (green) located at one end (Fig. 3a and b). The signal of CentO on this short chromosome was weaker than that on the normal chromosome 9 and the short chromosome also had two arms with different lengths (Fig. 3c and d). To further characterize this chromosome, a telomeric DNA probe, pAtT4 and chromosome 9-specific oligo probe were used for FISH (Fig. 3e and f). The telomeric probe signals (green) were located on the two ends of this short chromosome. These results suggested that both arms of this short chromosome were derived from chromosome 9 and one arm was the short arm of chromosome 9. The exact components of the short chromosome require further verification.

We detected 28 chromosomes in mutant YN6098 (Fig. 4), including two normal chromosome 9 and two additional small chromosomes with chromosome 9-specific signals. These two small chromosomes contained 45S rDNA signals at both ends (Fig. 4a and b). The CentO signals were located in the middle of these two chromosomes (Fig. 4c and d). These results showed that the small chromosomes are isochromosomes derived from the short arm of chromosome 9.

\section{Cross-species chromosome painting using the oligo probe}

To test the potential application of the chromosome 9 specific oligo probe on other genomes in the genus Oryza, we conducted a FISH assay using the chromosome 9-specific oligo and $45 \mathrm{~S}$ rDNA probes on the mitotic prometaphase chromosomes of $O$. eichingeri (CC, $2 \mathrm{n}=2 \mathrm{x}=24$ ). Distinct FISH signals of chromosome 9 and $45 \mathrm{~S}$ rDNA were detected on two and three chromosomes in each cell (Fig. 5a and b). For comparing chromosome 9 between $O$. eichingeri and O. sativa, the chromosome 9-specific oligo and 45S rDNA probes were utilized in a FISH experiment on mitotic prometaphase chromosomes which were prepared from the root tips of a triploid line (AAC, $2 n=3 \times=36$ ) derived from the $O$. eichingeri and a japonica rice, 02428. Distinct FISH signals were detected on three chromosomes in each cell (Fig. 5c and d). However, the FISH signals of the chromosome 9-specific oligo (red) and 45S rDNA 

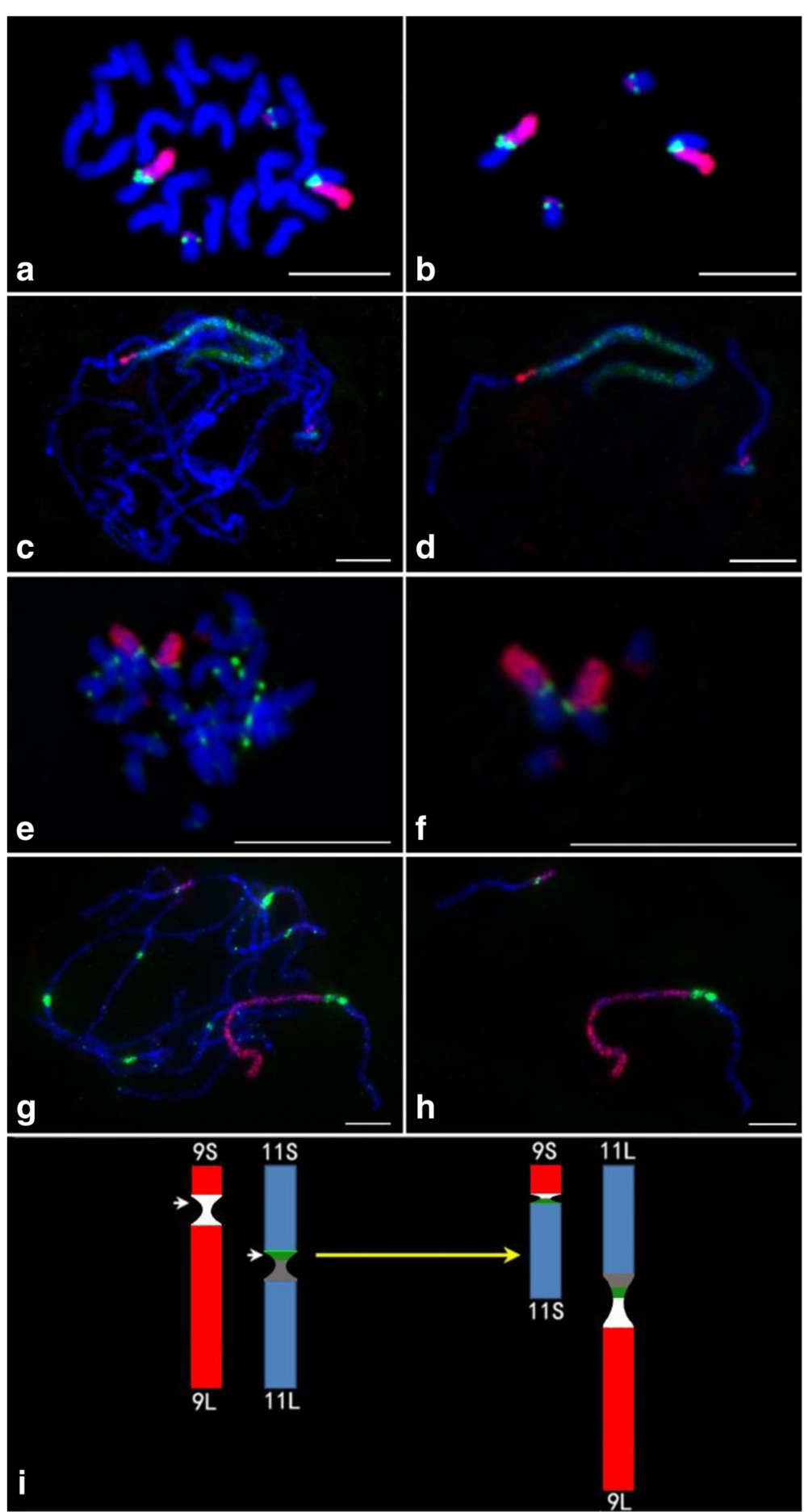

Fig. 2 Identification of a Chr9/11 translocation in an indica rice, Zhongxian 3037 by FISH. a The chromosome 9-specific (red) and 5S rDNA (green) probes on mitotic prometaphase chromosomes. c The chromosome 9-specific (green) and 5S rDNA (red) probes on chromosomes at the pachytene stage. The chromosome 9-specific (red) and CentO (green) probes fluorescing in chromosomes in the (e) mitotic prometaphase and (g) pachytene. $\mathbf{b}$ and $\mathbf{f}$ Two pairs of translocation chromosomes were digitally separated from the rest of the chromosomes shown in panels (a) and (e), respectively. $\mathbf{d}$ and $\mathbf{h}$ Two bivalents of the translocation chromosomes were digitally separated from the rest of the chromosomes shown in panels (c) and (g), respectively. Chromosomes were stained with DAPI. i The idiograms of chromosomes 9 and chromosome 11 of Zhongxian 3037 (left) and Chr9S.11S and Chr11 L.9 L of the translocation line (right). The white region is the centromere of chromosome 9. The green region is $5 \mathrm{~S} \mathrm{rDNA}$ and the gray region is the centromere of chromosome 11. White arrows indicate the breakpoints. Bars, $5 \mu \mathrm{m}$ 


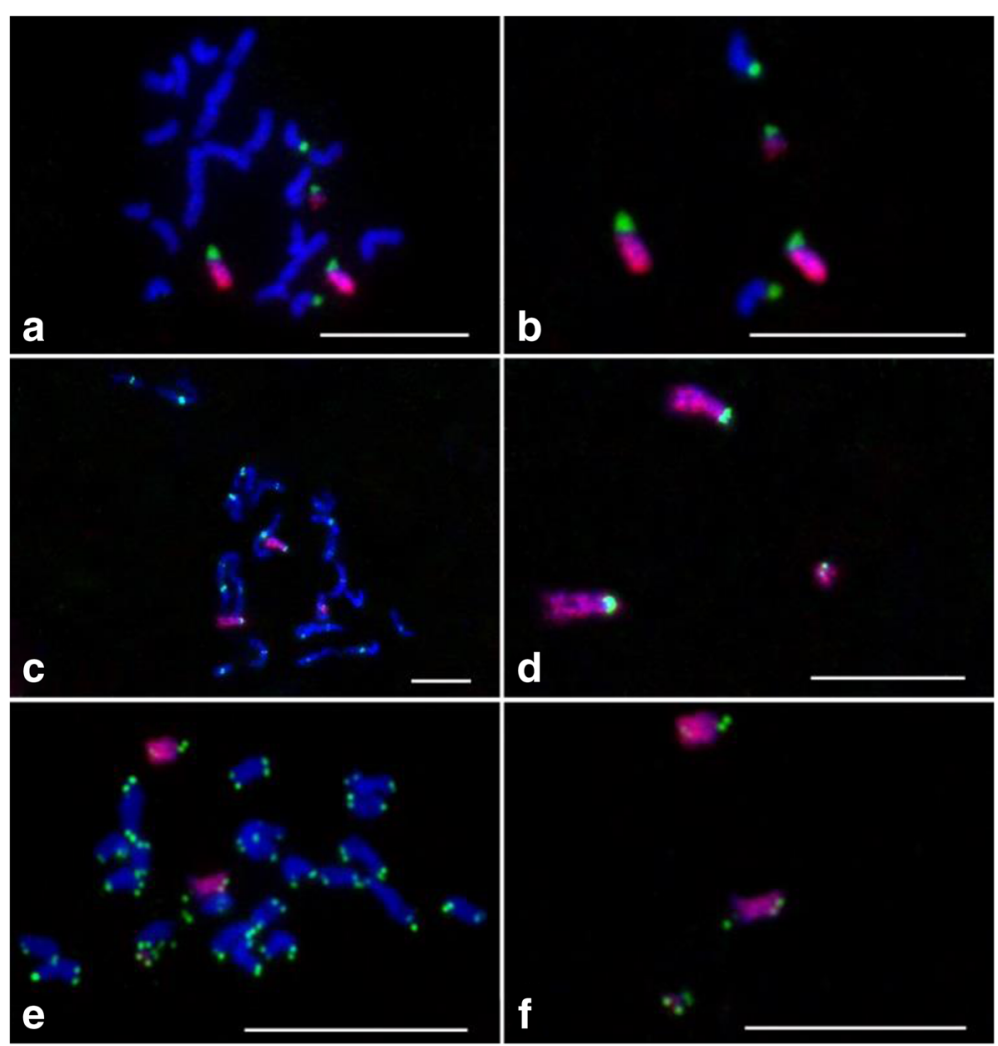

Fig. 3 Localization of chromosome 9-specific oligo library, CentO, pAtT4 and 45S rDNA probes on the chromosomes of aneuploid YN6077. a The chromosome 9-specific (red) and 45S rDNA (green) probes on mitotic prometaphase chromosomes. b Five chromosomes with 45S rDNA signals were digitally separated from the rest of the chromosomes shown in panel (a). c The chromosome 9-specific (red) and CentO (green) probes on mitotic prometaphase chromosomes. e The chromosome 9-specific (red) and pAtT4 (green) probes on mitotic prometaphase chromosomes. d and $\mathbf{f}$ Three chromosomes with chromosome 9-specific signals were digitally separated from the rest of the chromosomes shown in panels (c) and (e), respectively. Chromosomes were stained with DAPI. Bars, 5 um

(green) probes on one chromosome were weaker. To examine further the origins of the three chromosomes with chromosome 9 signal, the chromosome 9 specific oligo and CentO probes were used in FISH experiments (Fig. 5e and f). The chromosome with weaker chromosome 9 signal harbored no $\mathrm{CentO}$ signal and the CentO signals were observed on the two chromosomes with brighter chromosome 9 signals. These results indicated that the chromosome with weaker chromosome 9-specific signal is derived from O. eichingeri, as no CentO, a centromeric satellite on chromosome 9 of $O$. eichingeri [52]. And this chromosome 9 specific oligo probe can be used to track chromosome 9 of $O$. eichingeri.

\section{Discussion}

A chromosome-specific oligo probe offers a powerful way to identify a specific chromosome both in mitosis and meiosis in a Cucumis species [30]. Our experiment also demonstrated that the oligo probe based on the sequence of a japonica rice, can be used to identify chromosome 9 in both japonica and indica rice. This probe is also a useful tool to characterize the variations of related chromosomes. In this study, a chromosome 9-specific oligo set was used to characterize the variations of chromosome 9 in different mutants. This system appears to be more efficient and reliable than the traditional marker-based methods to detect chromosome variation. For example, it would be difficult to characterize the double isochromosome $9 \mathrm{~S}$ in line YN6089 (Fig. 4) using the traditional marker-based methods because the presence of six copies of $9 \mathrm{~S}$ in this line would complicate the interpretation of the marker-based data.

The two arms of the additional short chromosome had the signals of chromosome 9 in the mutant line, YN6077. However, the 45S rDNA signal was detected only at one end of this chromosome. The arm without $45 \mathrm{~S}$ rDNA in this chromosome is very short. We surmised a possible explanation for the occurrence of the short arm based on the fact that this mutant was derived from a trisomic 9. It may be a part of the long or short arm of chromosome 9. Moreover, a rearrangement involving the centromere may have also occurred in this 


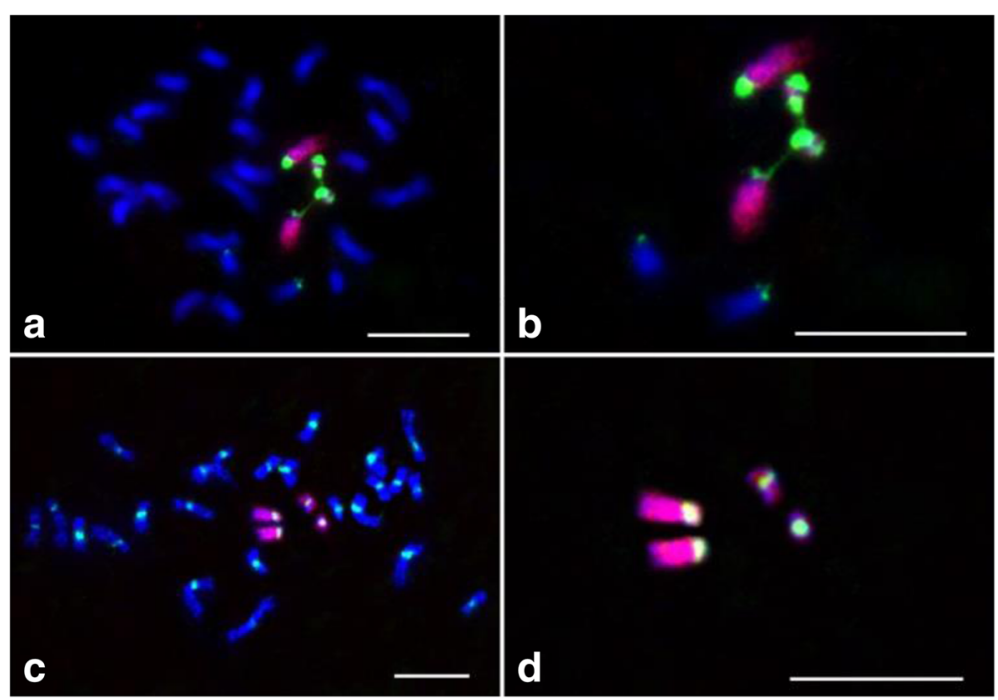

Fig. 4 FISH visualization of the localization of chromosome 9-specific oligo library, CentO, and $45 \mathrm{~S}$ rDNA probes on the chromosomes of aneuploid YN6089. a The chromosome 9-specific (red) and 45S rDNA (green) probes on mitotic prometaphase chromosomes. b Six chromosomes with 45S rDNA signals were digitally separated from the rest of the chromosomes shown in panel (a). c The chromosome 9specific (red) and CentO (green) probes on mitotic prometaphase chromosomes. d Four chromosomes with chromosome 9-specific signals were digitally separated from the rest of the chromosomes shown in panel (c). Chromosomes were stained with DAPI. Bars, $5 \mu \mathrm{m}$

short chromosome. To determine the components of this chromosome, chromosome 9 arm-specific or fragment-specific oligo probes may be used in future studies. Of course, as the technology on array-based comparative genomic hybridization continues to improve, we can continue to improve our ability to more rapidly and accurately identify chromosomal copy number variation in rice and other plant species $[53,54]$.

Oligo-based chromosome painting can potentially be used to detect chromosomes or chromosomal segments in germplasm stocks. This approach has been used successfully to paint homeologous chromosomes from several diploid and polyploidy Cucumis species that diverged from cucumber up to 12 million years ago. Pairing of a cucumber chromosome with a specific homeologous chromosome from a wild species can be monitored in the hybrid [30,31]. In the present study, chromosome 9-specific probe based on a japonica rice also exhibited similar signals for chromosome 9 in $O$. eichingeri ( $\mathrm{C}$ genome). This indicates that sequence similarity exists in chromosome 9 between $O$. sativa and $O$. eichingeri. It is consistent with the result that $O$. sativa and $O$. officinalis share a high degree of synteny of RFLP markers [55]. However, the FISH signals of both oligo probes and 45S rDNA on chromosome 9 in $O$. eichingeri were much weaker than those in $O$. sativa, indicating that some differentiation has been occurred between O. eichingeri and O. sativa [35]. Thus, this chromosome specific oligo probe can be used to track homeologous chromosome pairing in the hybrids derived from $O$. sativa and $O$. eichingeri. However, whether this probe would be able to efficiently distinguish chromosome 9 among other rice genomes and monitor the homeologous chromosome pairing in their heterogeneous hybrids remains elusive.

\section{Conclusions}

The chromosome-specific probe is a fundamental tool of chromosome painting. We developed a pooled chromosome 9-specific probe in rice, which contains 25,000 oligos based on the genome sequence of a japonica rice $(O$. sativa, $\mathrm{AA}, 2 \mathrm{n}=2 \mathrm{x}=24$ ). Chromosome 9 was easily identified in both japonica and indica rice using this chromosome 9-painting probe. The probe was also successfully used to identify and characterize chromosome 9 in additional lines of $O$. sativa, a translocation line, two new aneuploids associated with chromosome 9 and a wild rice $(O$. eichingeri, $C C, 2 n=2 \times=24)$. The study reveals that a pool of oligos specific to a chromosome is a useful tool for chromosome painting in rice.

\section{Methods}

\section{Plant materials}

A total of six kinds of rice were used in this study: one cultivar of japonica rice, Nipponbare (O. sativa, AA, $2 \mathrm{n}$ $=2 \mathrm{x}=24$ ); two aneuploids derived from the progeny of a trisomic 9 of the indica rice Zhongxian 3037 (O. sativa); a translocation line with reciprocal translocation between chromosomes 9 and 11 in Zhongxian 3037 [48]; a wild rice, $O$. eichingeri $(\mathrm{CC}, 2 \mathrm{n}=2 \times=24)$ and a triploid (AAC, $2 \mathrm{n}=3 \times=36)$ derived from O. eichingeri $(\mathrm{CC}, 2 \mathrm{n}$ $=2 \times=24)$ and a japonica rice 02428 [45]. All materials 

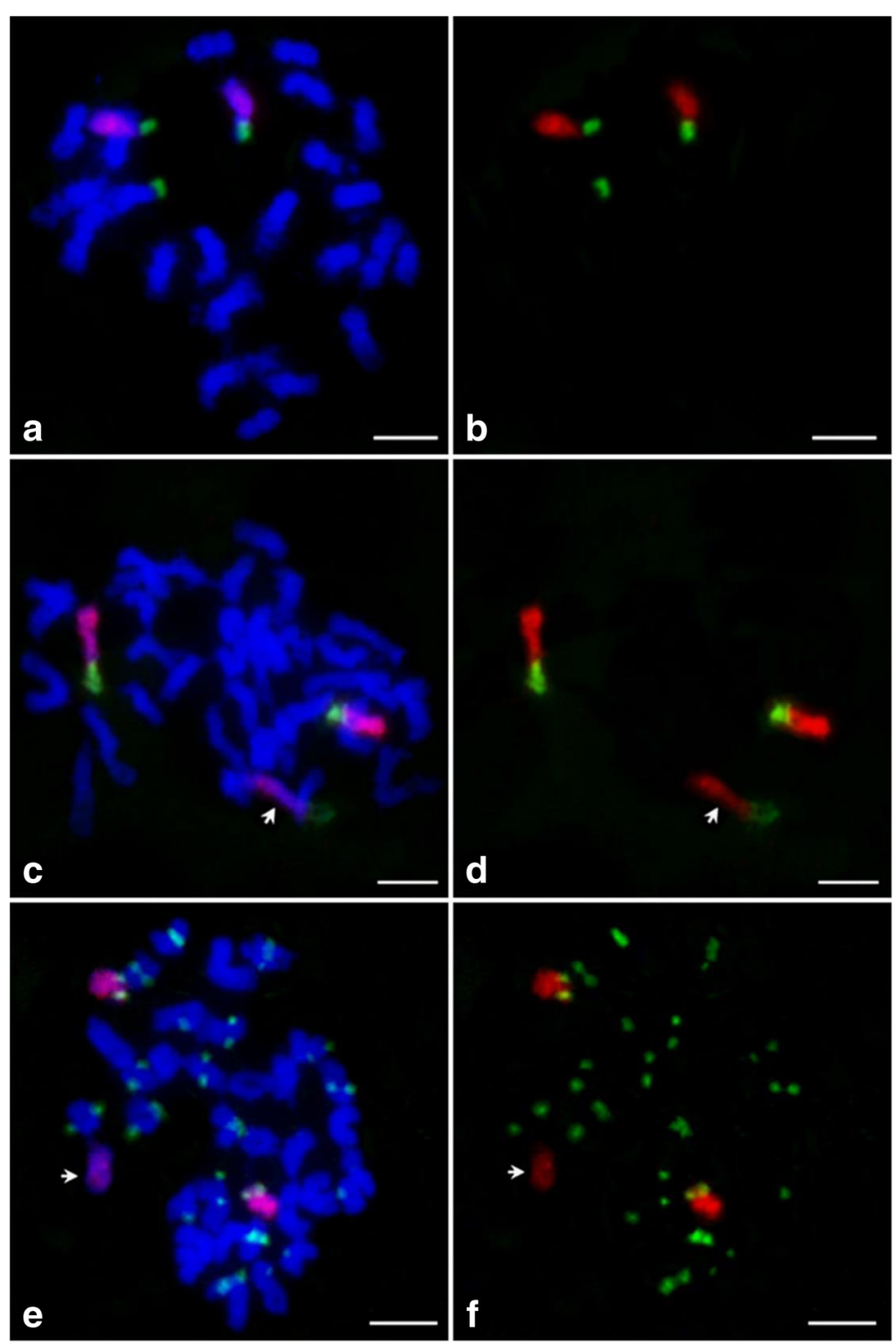

Fig. 5 Localization of the chromosome 9-specific oligo library, $45 \mathrm{~S}$ rDNA and CentO probes on the chromosomes of 0 . eichingeri and a triploid (AAC). The chromosome 9-specific (red) and 45S rDNA probes (green) fluorescing in prometaphase chromosomes of the (a) O. eichingeri (CC, $2 \mathrm{n}$ $=2 x=24$ ) and $(\mathbf{c})$ the triploid (AAC, $2 n=3 x=36$ ) derived from 0 . eichingeri and 02428. $\mathbf{b}$ and $\mathbf{d}$ The chromosome 9 -specific and $45 \mathrm{~S} r \mathrm{rNA}$ signals were digitally separated from the rest of the chromosomes in panels (a) and (c), respectively. e The chromosome 9-specific (red) and CentO (green) probes on chromosomes of the triploid. $\mathbf{f}$ The chromosome 9-specific and CentO signals were digitally separated from the rest of the chromosomes in (e). White arrows indicate the chromosome 9 from O. eichingeri. Chromosomes were stained with DAPI. Bars, $5 \mu \mathrm{m}$

were planted in the trial fields of Yangzhou University (Yangzhou, Jiangsu Province, China).

Root tips were harvested from rice plants and pretreated in $0.002 \mathrm{M}$ 8-hydroxyquinoline at $20^{\circ} \mathrm{C}$ for $2 \mathrm{~h}$, then fixed in methanol-acetic acid (3:1) and stored at $20{ }^{\circ} \mathrm{C}$ until use. Squashes of root tips were prepared according to $\mathrm{Yu}$ et al. [56]. Young panicles of rice plants were harvested and fixed in Carnoy's solution (ethanol: glacial acetic acid, 3: 1) and stored at $-20{ }^{\circ} \mathrm{C}$. Squashes of panicles were prepared in acetic-carmine solution according to Cheng et al. [57].

\section{Bioinformatics pipeline for oligo selection}

We used the Chorus software (https://github.com/forrestzhang/Chorus) for selecting chromosome 9-specific oligos [30]. In general, we used the rice genome (TIGR7, http://rice.plantbiology.msu.edu/) as reference [58]. All repeat sequences of chromosome 9 were filtered by applying RepeatMasker (http://www.repeatmasker.org). We divided repeat filtered chromosome 9 sequences into $45 \mathrm{nt}$ with a step size of $5 \mathrm{nt}$. Then we mapped all the oligo sequences to the whole genome of rice and filtered oligos which can map to two or more loci with $75 \%$ 
homology. Next, we calculated the melting temperature (Tm) and hairpin Tm of each oligo. Oligos with $\mathrm{dTm}>10$ $(\mathrm{dTm}=\mathrm{Tm}$-hairpin $\mathrm{Tm})$ were kept to build a probe set.

\section{Probe preparation and FISH}

The oligo library was synthesized by MYcroarray (Ann Arbor, MI, USA). Probe preparation from the synthesized oligo library was conducted as described by Han et al. [30]. FISH analysis was performed as described by Han et al. [30]. The biotin- or digoxigenin-labeled single-stranded oligos prepared from the library were directly used as FISH probes. CentO which contains a 155-bp satellite repeat of a rice centromere [50], a pTa794 clone containing the coding sequence for the $5 \mathrm{~S}$ rDNA of wheat [59], 45S rDNA [47] and a pAtT4 clone containing $A$. thaliana telomeric DNA [60] were labeled with either digoxigenin-11-dUTP or biotin-dUTP (Roche) by standard nick translation and included in FISH probes [61].

Each probe was detected using a fluorescein isothiocyanate conjugated anti-biotin or anti-digoxigenin antibody (Vector Laboratories). The chromosomes were counterstained with 4', 6' -diamidino-phenylindole (DAPI) in Vectashield antifading solution (Vector laboratories). Chromosomes and FISH signals were observed under an Olympus BX61 fluorescence microscope and images were captured with a DVC1412 CCD camera using IPLab software.

\section{Additional file}

Additional file 1: The chromosome 9-specific oligo sequence data. (BED $1827 \mathrm{~kb}$ )

\section{Abbreviations}

45S rDNA: 455 ribosomal RNA gene; 55 rDNA: 55 ribosomal RNA gene; BAC: Bacterial artificial chromosome; CP: Chromosome painting; DAPI: 4', 6'diamidino-phenylindole; FISH: Fluorescence in situ hybridization; oligos: Oligonucleotides; Tm: Melting temperature; YAC: Yeast artificial chromosomes

\section{Acknowledgements}

This work was supported by grants from the National Key Research and Development Program (2016YFD0102001-006), the National Natural Science Foundation of China (31670313), Key Project of Jiangsu Education Department of China (15KJA180010), Yangzhou City Science and Technology Plan (YZ2017059), and the Priority Academic Program Development of Jiangsu Higher Education Institutions (PAPD)

\section{Availability of data and materials}

The data sets supporting the results of this article are included within the article.

\section{Authors' contributions}

$H Y, J J$ and MG conceived the project and designed the experiments; LH, MX, TZ, ZX, WW, JZ, WJ, MY, CZ and ZG performed the experiments, planted materials and collected samples. LH wrote the paper. All authors read and approved the final manuscript.
Ethics approval and consent to participate

Not applicable.

\section{Competing interests}

The authors declare that they have no competing interests.

\section{Author details}

${ }^{1}$ Key Laboratory of Plant Functional Genomics of Ministry of Education/ Jiangsu Key Laboratory of Crop Genetics and Physiology/Co-Innovation Center for Modern Production Technology of Grain Crops, Yangzhou University, Yangzhou 225009, China. ${ }^{2}$ Department of Horticulture, University of Wisconsin-Madison|, Madison, WI 53706, USA.

Received: 2 December 2017 Accepted: 24 May 2018

Published online: 07 June 2018

\section{References}

1. Pinkel D, Landegent J, Collins C, Fuscoe J, Segraves R, Lucas J, Gray J. Fluorescence in situ hybridization with human chromosome-specific libraries: detection of trisomy 21 and translocations of chromosome 4. Proc Natl Acad Sci U S A. 1988;85(23):9138-42.

2. Ried T, Schröck E, Ning Y, Wienberg J. Chromosome painting: a useful art Hum Mol Genet. 1998;7(10):1619-26.

3. Rens W, Fu B, O'brien PC, Ferguson-Smith M. Cross-species chromosome painting. Nat Protoc. 2006;1998(2):783

4. Graphodatsky AS, Trifonov VA, Roscoe S. The genome diversity and karyotype evolution of mammals. Mol Cytogenet. 2011;4(1):22.

5. Cremer M, Grasser F, Lanctôt C, Müller S, Neusser M, Zinner R, Solovei I, Cremer T. Multicolor 3D fluorescence in situ hybridization for imaging interphase chromosomes. The Nucleus: Volume 1: Nuclei and Subnuclear Components. 2008:463:205-39.

6. Cremer T, Kurz A, Zirbel R, Dietzel S, Rinke B, Schröck E, Speicher MR, Mathieu U, Jauch A, Emmerich P. Role of chromosome territories in the functional compartmentalization of the cell nucleus. Cold Spring Harb Symp Quant Biol. 1993:58(534):777-92.

7. Kurz A, Lampel S, Nickolenko JE, Bradl J, Benner A, Zirbel RM, Cremer T, Lichter P. Active and inactive genes localize preferentially in the periphery of chromosome territories. J Cell Biol. 1996;135(5):1195-205.

8. Müller S, Stanyon R, O'Brien PC, Ferguson-Smith MA, Plesker R, Wienberg J. Defining the ancestral karyotype of all primates by multidirectional chromosome painting between tree shrews, lemurs and humans. Chromosoma. 1999:108(6):393-400.

9. Wienberg J, Jauch A, Stanyon R, Cremer T. Molecular cytotaxonomy of primates by chromosomal in situ suppression hybridization. Genomics. 1990;8(2):347-50.

10. Marshall R, Obe G. Application of chromosome painting to clastogenicity testing in vitro. Environ Mol Mutagen. 1998;32(3):212-22.

11. Gray JW, Pinkel D. Molecular cytogenetics in human cancer diagnosis. Cancer. 1992;69(S6):1536-42.

12. Carter NP, Bebb CE, Nordenskjo M, Ponder BA, Tunnacliffe A. Degenerate oligonucleotide-primed PCR: general amplification of target DNA by a single degenerate primer. Genomics. 1992;13(3):718-25.

13. Yang F, O'Brien P, Milne B, Graphodatsky A, Solanky N, Trifonov V, Rens W Sargan D, Ferguson-Smith M. A complete comparative chromosome map for the dog, red fox, and human and its integration with canine genetic maps. Genomics. 1999;62(2):189-202

14. Ferguson-Smith MA, Trifonov V. Mammalian karyotype evolution. Nat Rev Genet. 2007:8(12):950-62

15. Idziak D, Betekhtin A, Wolny E, Lesniewska K, Wright J, Febrer M, Bevan MW Jenkins G, Hasterok R. Painting the chromosomes of Brachypodium - current status and future prospects. Chromosoma. 2011;120(5):469-79.

16. Jiang J, Gill BS. Current status and the future of fluorescence in situ hybridization (FISH) in plant genome research. Genome. 2006:49(9):1057-68.

17. Lysak MA, Mandáková T. Analysis of plant meiotic chromosomes by chromosome painting. Methods Mol Biol. 2013;990(2):13-24.

18. Schubert I, Fransz PF, Fuchs J, de Jong JH. Chromosome painting in plants. Methods Cell Sci. 2001;23(1-3):57-69.

19. Fransz PF, Armstrong S, de Jong JH, Parnell LD, van Drunen C, Dean C Zabel P, Bisseling T, Jones GH. Integrated cytogenetic map of chromosome arm $4 S$ of $A$. thaliana: structural organization of heterochromatic knob and centromere region. Cell. 2000;100(3):367-76. 
20. Lysak MA, Fransz PF, Ali HB, Schubert I. Chromosome painting in Arabidopsis thaliana. Plant J. 2001;28(6):689-97.

21. Betekhtin A, Jenkins $G$, Hasterok R. Reconstructing the evolution of Brachypodium genomes using comparative chromosome painting. PLoS One. 2014;9(12):e115108

22. Lysak MA, Berr A, Pecinka A, Schmidt R, McBreen K, Schubert I. Mechanisms of chromosome number reduction in Arabidopsis thaliana and related Brassicaceae species. Proc Natl Acad Sci U S A. 2006;103(13):5224-9.

23. Lysak MA, Koch MA, Pecinka A, Schubert I. Chromosome triplication found across the tribe Brassiceae. Genome Res. 2005;15(4):516-25.

24. Mandáková T, Joly S, Krzywinski M, Mummenhoff K, Lysak MA. Fast diploidization in close mesopolyploid relatives of Arabidopsis. Plant Cell. 2010;22(7):2277-90.

25. Mandáková T, Lysak MA. Chromosomal phylogeny and karyotype evolution in $X=7$ crucifer species (Brassicaceae). Plant Cell. 2008;20(10):2559-70.

26. Lou Q, Zhang Y, He Y, Li J, Jia L, Cheng C, Guan W, Yang S, Chen J. Singlecopy gene-based chromosome painting in cucumber and its application for chromosome rearrangement analysis in Cucumis. Plant J. 2014;78(1):169-79.

27. Boyle S, Rodesch MJ, Halvensleben HA, Jeddeloh JA, Bickmore WA. Fluorescence in situ hybridization with high-complexity repeat-free oligonucleotide probes generated by massively parallel synthesis. Chromosom Res. 2011;19(7):901-9.

28. Yamada NA, Rector LS, Tsang P, Carr E, Scheffer A, Sederberg MC, Aston ME, Ach RA, Tsalenko A, Sampas N. Visualization of fine-scale genomic structure by oligonucleotide-based high-resolution FISH. Cytogenetic \& Genome Research. 2011;132(4):248-54.

29. Beliveau BJ, Joyce EF, Apostolopoulos N, Yilmaz F, Fonseka CY, Mccole RB, Chang Y, Li JB, Senaratne TN, Williams BR. Versatile design and synthesis platform for visualizing genomes with Oligopaint FISH probes. Proc Natl Acad Sci U S A. 2012;109(52):21301-6.

30. Han Y, Zhang T, Thammapichai P, Weng Y, Jiang J. Chromosome-specific painting in Cucumis species using bulked oligonucleotides. Genetics. 2015; 200(3):771-9.

31. Li K, Wang H, Wang J, Sun J, Li Z, Han Y. Divergence between C. melo and african Cucumis species identified by chromosome painting and rDNA distribution pattern. Cytogenetic and genome research. 2016;150(2):150-5.

32. Xin H, Zhang T, Han Y, Wu Y, Shi J, Xi M, Jiang J. Chromosome painting and comparative physical mapping of the sex chromosomes in Populus tomentosa and Populus deltoides. Chromosoma. 2018; https://doi.org/10. 1007/s00412-018-0664-y.

33. Braz GT, He L, Zhao H, Zhang T, Semrau K, Rouillard JM, Torres GA, Jiang J. Comparative oligo-FISH mapping: an efficient and powerful methodology to reveal karyotypic and chromosomal evolution. Genetics. 2018;208(2):513-23.

34. Ge S, Sang T, Lu BR, Hong DY. Phylogeny of rice genomes with emphasis on origins of allotetraploid species. Proc Natl Acad Sci U S A. 1999;96(25):14400-5.

35. Vaughan DA, Morishima H, Kadowaki K. Diversity in the Oryza genus. Curr Opin Plant Biol. 2003;6(2):139-46.

36. Aggarwal RK, Brar DS, Khush GS. Two new genomes in the Oryza complex identified on the basis of molecular divergence analysis using total genomic DNA hybridization. Mol Gen Genet. 1997;254(1):1-12.

37. Oka HI, Chang WT. The impact of cultivation on populations of wild rice, Oryza sativa f. spontanea. Phyton. 1959;13:105-77.

38. Oka HI, Chang WT. Rice varieties intermediate between wild and cultivated forms and the origin of the japonica type. Bot Bull Acad Sin. 1962;3:109-31.

39. Rakshit S, Rakshit A, Matsumura H, Takahashi Y, Hasegawa Y, Ito A, Ishii T, Miyashita NT, Terauchi R. Large-scale DNA polymorphism study of Oryza sativa and 0 . rufipogon reveals the origin and divergence of Asian rice. Theoretical \& Applied Genetics. 2007;1 14(4):731-43.

40. Huang X, Kurata N, Wei X, Wang ZX, Wang A, Zhao Q, Zhao Y, Liu K, Lu H, $\mathrm{Li}$ W. A map of rice genome variation reveals the origin of cultivated rice. Nature. 2012;490(7421):497-501

41. Aggarwal RK, Brar DS, Nandi S, Huang N, Khush GS. Phylogenetic relationships among Oryza species revealed by AFLP markers. Theoretical \& Applied Genetics. 1999;98(8):1320-8.

42. He RF, Wang Y, Shi Z, Ren X, Zhu L, Weng Q, He GC. Construction of a genomic library of wild rice and Agrobacterium-mediated transformation of large insert DNA linked to BPH resistance locus. Gene. 2003;321:113-21.

43. Huang Z, He G, Shu L, Li X, Zhang Q. Identification and mapping of two brown planthopper resistance genes in rice. Theor Appl Genet. 2001;102(6):929-34.

44. Tan GX, Ren X, Weng QM, Shi ZY, Zhu LL, He GC. Mapping of a new resistance gene to bacterial blight in rice line introgressed from Oryza officinalis. Acta Genet Sin. 2004;31(7):724-9.
45. Yan H, Xiong Z, Min S, Hu H, Zhang Z, Tian S, Tang S. The transfer of brown planthopper resistance from Oryza eichingeri to O sativa. Acta Genetica Sinica. 1997;24(5):424-31.

46. Goff SA, Ricke D, Lan T-H, Presting G, Wang R, Dunn M, Glazebrook J, Sessions A, Oeller P, Varma H. A draft sequence of the rice genome (Oryza sativa L. ssp. japonica). Science. 2002;296(5565):92-100.

47. Cheng Z, Yang X, Yu H, Gu M. A study of the number of SAT-chromosome in rice. Acta Genet Sin. 1997;25(3):225-31.

48. Wang G, Li H, Cheng Z, Jin W. A novel translocation event leads to a recombinant stable chromosome with interrupted centromeric domains in rice. Chromosoma. 2013:122(4):295-303.

49. Kamisugi Y, Nakayama S, Nakajima R, Ohtsubo H, Ohtsubo E, Fukui K. Physical mapping of the 5S ribosomal RNA genes on rice chromosome 11. Mol Gen Genet. 1994;245(2):133.

50. Cheng Z, Dong F, Langdon T, Ouyang S, Buell CR, Gu M, Blattner FR, Jiang J. Functional rice centromeres are marked by a satellite repeat and a centromere-specific retrotransposon. Plant Cell. 2002;14(8):1691-704.

51. Cheng Z, Yan H, Yu H, Tang S, Jiang J, Gu M, Zhu L. Development and applications of a complete set of rice telotrisomics. Genetics. 2001;157(1): 361-8.

52. Bao W, Zhang W, Yang Q, Zhang Y, Han B, Gu M, Xue Y, Cheng Z. Diversity of centromeric repeats in two closely related wild rice species, Oryza officinalis and Oryza rhizomatis. Mol Genet Genomics. 2006;275(5):421-30.

53. Smida J, Xu H, Zhang Y, Baumhoer D, Ribi S, Kovac M, Von Luettichau I, Bielack S, O'Leary VB, Leib-Mösch C. Genome-wide analysis of somatic copy number alterations and chromosomal breakages in osteosarcoma. Int $J$ Cancer. 2017;141(4):816.

54. Wang W, Wang C, Dawson DB, Thorland EC, Lundquist PA, Eckloff BW, Wu Y, Baheti S, Evans JM, Scherer SS. Target-enrichment sequencing and copy number evaluation in inherited polyneuropathy. Neurology. 2016;86(19): 1762.

55. Jin H, Tan G, Brar DS, Tang M, Li G, Zhu L, He G. Molecular and cytogenetic characterization of an Oryza officinalis-O. sativa chromosome 4 addition line and its progenies. Plant Mol Biol. 2006;62(4-5):769-77.

56. Yu H, Gong Z, Su Y, Gu M. Isolation and identification of the three rice monotelosomics. Chin Sci Bull. 2005;50(19):2182-6.

57. Cheng Z, Buell CR, Wing RA, Gu M, Jiang J. Toward a cytological characterization of the rice genome. Genome Res. 2001;11(12):2133-41.

58. Kawahara Y, Bastide MDL, Hamilton JP, Kanamori H, Mccombie WR, Shu O, Schwartz DC, Tanaka T, Wu J, Zhou S. Improvement of the Oryza sativa Nipponbare reference genome using next generation sequence and optical map data. Rice. 2013;6(1):3-10.

59. Gerlach WL, Dyer TA. Sequence organization of the repeating units in the nucleus of wheat which contain $5 S$ rRNA genes. Nucleic Acids Res. 1980; 8(21):4851-65.

60. Richards EJ, Ausubel FM. Isolation of a higher eukaryotic telomere from Arabidopsis thaliana. Cell. 1988;53(1):127-36.

61. Dong F, Miller JT, Jackson SA, Wang GL, Ronald PC, Jiang J. Rice (Oryza sativa) centromeric regions consist of complex DNA. Proc Natl Acad Sci U S A. 1998;95(14):8135-40.

Ready to submit your research? Choose BMC and benefit from

- fast, convenient online submission

- thorough peer review by experienced researchers in your field

- rapid publication on acceptance

- support for research data, including large and complex data types

- gold Open Access which fosters wider collaboration and increased citations

- maximum visibility for your research: over $100 \mathrm{M}$ website views per year

At BMC, research is always in progress.

Learn more biomedcentral.com/submissions 